\title{
Social encounter profiles of greater Melbourne residents, by location - a telephone survey
}

David A. Rolls ${ }^{1+}$, Nicholas L. Geard ${ }^{2 \dagger}$, Deborah J. Warr ${ }^{3}$, Paula M. Nathan ${ }^{2}$, Garry L. Robins ${ }^{1}$, Philippa E. Pattison ${ }^{1}$, James M. McCaw ${ }^{2,4,5}$ and Jodie McVernon ${ }^{2,5^{*}}$

\begin{abstract}
Background: Models of infectious disease increasingly seek to incorporate heterogeneity of social interactions to more accurately characterise disease spread. We measured attributes of social encounters in two areas of Greater Melbourne, using a telephone survey.

Methods: A market research company conducted computer assisted telephone interviews (CATIs) of residents of the Boroondara and Hume local government areas (LGAs), which differ markedly in ethnic composition, age distribution and household socioeconomic status. Survey items included household demographic and socio-economic characteristics, locations visited during the preceding day, and social encounters involving two-way conversation or physical contact. Descriptive summary measures were reported and compared using weight adjusted Wald tests of group means.
\end{abstract}

Results: The overall response rate was $37.6 \%$, higher in Boroondara $[n=650,(46 \%)]$ than Hume $[n=657(32 \%)]$. Survey conduct through the CATI format was challenging, with implications for representativeness and data quality. Marked heterogeneity of encounter profiles was observed across age groups and locations. Household settings afforded greatest opportunity for prolonged close contact, particularly between women and children. Young and middle-aged men reported more age-assortative mixing, often with non-household members. Preliminary comparisons between LGAs suggested that mixing occurred in different settings. In addition, gender differences in mixing with household and non-household members, including strangers, were observed by area.

Conclusions: Survey administration by CATI was challenging, but rich data were obtained, revealing marked heterogeneity of social behaviour. Marked dissimilarities in patterns of prolonged close mixing were demonstrated by gender. In addition, preliminary observations of between-area differences in socialisation warrant further evaluation.

Keywords: Social networks, Socioeconomic factors, Population characteristics

\footnotetext{
* Correspondence: j.mcvernon@unimelb.edu.au

${ }^{\dagger}$ Equal contributors

${ }^{2}$ Modelling and Simulation Unit, Centre for Epidemiology and Biostatistics,

Melbourne School of Population and Global Health, The University of

Melbourne, Melbourne, Australia

${ }^{5}$ Modelling and Simulation Unit, Infection and Immunity Theme, Murdoch

Childrens Research Institute, Parkville, Australia

Full list of author information is available at the end of the article
}

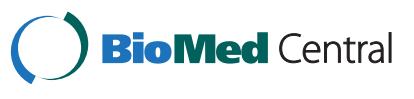

(c) 2015 Rolls et al. Open Access This article is distributed under the terms of the Creative Commons Attribution 4.0 International License (http://creativecommons.org/licenses/by/4.0/), which permits unrestricted use, distribution, and reproduction in any medium, provided you give appropriate credit to the original author(s) and the source, provide a link to the Creative Commons license, and indicate if changes were made. The Creative Commons Public Domain Dedication waiver (http://creativecommons.org/publicdomain/zero/1.0/) applies to the data made available in this article, unless otherwise stated. 


\section{Background}

Models of infectious disease epidemiology are becoming ever more complex in their construction, in recognition of the importance of heterogeneity of individuals and their social interactions to transmission of infection [1]. Such models have been informed by the increasing acquisition of data in a range of studies, describing patterns of human interaction at the level of age group [2], within social networks [3], or related to movements in geographical space and time [4].

In earlier work, we piloted methods to acquire data on social encounters of individuals within the locations visited over the course of several days, comparing anticipated contacts with prospective records entered either in a paper diary (modified from European study instruments) or portable electronic device $[5,6]$. While that study provided rich and detailed information, the scope of data collection was necessarily limited by budgetary and logistic constraints associated with the requirement for face-to-face recruitment and training, making detailed characterization of large population samples challenging.

In this project, we are seeking to document the influence of individual and area-level factors on social encounter profiles, which have been qualitatively observed to influence perceived social network characteristics [7]. In particular, individuals from less advantaged neighbourhoods have been described as likely to have close local networks, and fewer 'bridging' ties beyond their immediate area in comparison with counterparts in socio-economically advantaged areas who are more likely to have broadly distributed connections [7]. This project seeks to define in more detail the attributes of social encounters, beyond degree distribution, that might quantifiably capture such differences.

We recruited residents of two local government areas of metropolitan Melbourne (the state capital of Victoria, Australia) that differ markedly in terms of demographics and socio-economic status as 'proof of concept' of such influence. In order to reach as large and broadly representative a sample as possible, we employed telephonebased survey methods for recruitment and collection of encounter data. This paper reports on our experience of this survey approach, and initial findings summarising encounters in the two regions of interest.

\section{Methods}

\section{Study population}

The study recruited participants from two local government areas (LGAs) in greater Melbourne between January and April 2013 (Fig. 1). Each area had a similar population size but markedly different population characteristics, as outlined below.
Hume is a growing fringe municipality $20 \mathrm{kms}$ from Melbourne's central business district (CBD), retaining a rural character in its North. $43 \%$ of its population was born overseas with the most frequent countries of origin being Turkey (5\%) and Iraq (5\%), and $31 \%$ of residents were less than 18 years of age according to the 2011 census [8]. Median household income for families with children was around AUD $\$ 1,300$ per week [8]. In contrast, Boroondara incorporates a number of established inner-eastern suburbs, $5 \mathrm{kms}$ east of the CBD, bounded by rivers and parkland. While $34 \%$ of its residents were born overseas, the most common countries of origin were China (5\%) and the United Kingdom (3\%), and only $21 \%$ were less than 18 years old in 2011 [9]. Median household income for families with dependent children at that time exceeded AUD\$2,500 per week [9]. Further details of the demographic and socioeconomic characteristics of each LGA can be found in the Additional file 1: Cleaning, Geocoding and Weighting.

\section{Survey methods}

A market research company was contracted to conduct computer assisted telephone interviews (CATI) of 1000 respondents in each LGA to characterize social encounter profiles in locations visited over the course of a single day. Random digit dialling within local telephone exchanges was used to identify the sample, in order to avoid selection bias for longer-term resident individuals associated with the use of listed numbers (as short term tenants may change land lines frequently), as well as residents choosing to withhold their details from public listing. Unfortunately, individuals with only mobile phone or voice over internet protocol telephone access could not be included in the frame as it was not possible to assign such numbers to a residential location within the LGA boundaries. Respondents in Hume were given the option of completing the survey in either English or Turkish, given the high proportion (5\%) of Turkish born residents in that LGA, but resources were not available to offer interviews in other languages.

During a telephone interview (approximately 20-30 min in length), participants were asked to describe the basic demographic characteristics of their household including the number, age and occupational status of family members and key indicators of household economic status, including housing tenure. They were then asked to sequentially list all locations visited and movements between those locations on the previous day, including the type of location (e.g., home, work, retail, private transport) and when they left the location. They were also asked to list all social encounters with an individual or defined group (e.g., school class, workplace contacts, church congregation) for ease of reporting in each location. Encounters were defined as a two-way 


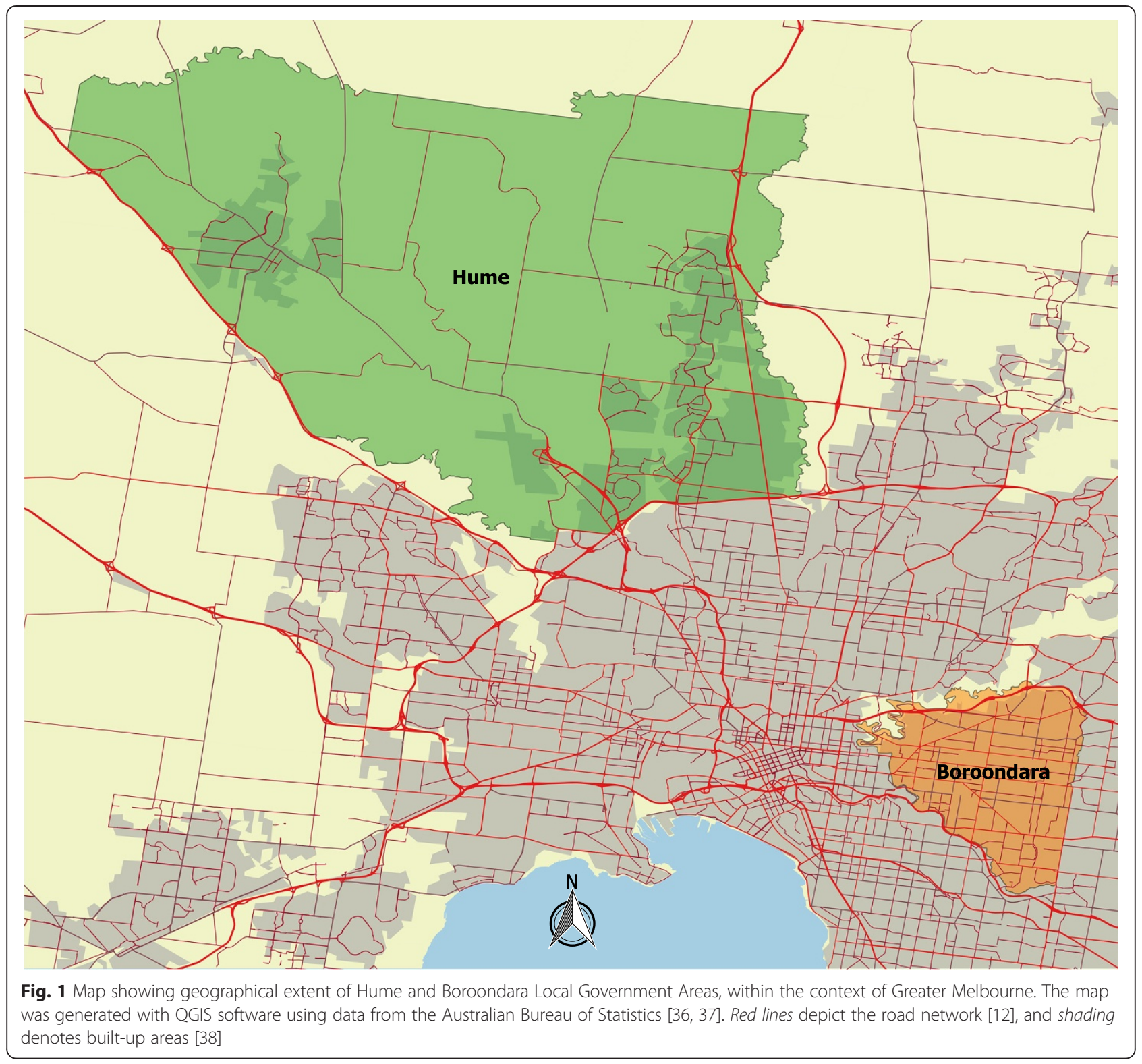

face-to-face conversation of more than three words or any physical contact. All interviews with participants were conducted by telephone and entered into an electronic form by employees of the market research company. Survey instruments are provided in Additional file 2: Questionnaire and Additional file 3: Contact diary.

\section{Data preparation}

Addresses of visited locations were checked for accuracy and completeness, and corrected to a standard format. Where necessary, place name descriptions were assigned street addresses with reference to corporate websites (retail locations) and/or publicly available searchable mapping tools. Time data were similarly checked for logical order and consistency, and calculated in relation to expected travel time between locations as necessary for confirmation. Further details of standard procedures followed for data cleaning are provided in Additional file 1 (Section 2 - Address Accuracy and Cleaning and Section 3 - Time Consistency and Cleaning).

\section{Geocoding}

Addresses were geocoded using a mix of API queries including Bing Maps [10], Mapquest [11] and OpenStreetMaps [12], and manually via the Google Maps website [13]. MATLAB 7.14 was used to script all work. Rules for geocoding, location matching and confirmation of accuracy are described in more detail in Additional file 1 (Section 4 - Geocoding). 


\section{Weighting}

Demographic and socio-economic characteristics of the LGAs under study were obtained from the Australian Bureau of Statistics 2011 census using publicly available methods [14]. Iterative proportional fitting (i.e., raking) was used to determine sampling weights to reduce the effects of sample bias. Raking weights were computed and applied to the survey data using STATA 10 with reference to the following population descriptors.

1. Joint distribution of five age categories and gender (10 categories);

2. Marital status (coupled, never married, other);

3. Australian born (yes/no);

4. Household type (couple with children, couple without children, one parent family, other family, lone person household, other household);

5. Household size (1, 2, 3, 4, 5, 6+);

6. Home ownership (owned outright, owned with a mortgage, renting, other, missing).

Large weights were truncated at 7 (Boroondara) and 6 (Hume) to remove extreme weights. These cutoffs were the smallest values for which goodness-of-fit tests comparing raked variables with census totals did not reject at the $5 \%$ level. Additional details and justification of the weighting procedure are available in Additional file 1 (Section 5 - Biased Sampling, Raking and Sampling Weights). In particular, goodness-of-fit results comparing the sample with the 2011 census both before and after raking are shown in Table S7 of Additional file 1.

\section{Analysis of social encounters}

Summary measures of recorded encounters with individuals and groups were characterised for comparison with our own and other previous studies, including separate description of the subset involving any physical contact as a proxy measure of intensity. The number and duration of contacts was reported by location type. Interactions within and between age groups were considered separately for men and women. Social heterogeneity was further assessed, by differentiating between contacts with household members, known individuals and strangers. The influence of household size on the number of encounters within and beyond the household unit was considered. These various measures were tabulated by LGA of residence, and differences between regions assessed using weight-adjusted Wald tests of the difference between group means.

\section{Ethical approvals}

The study protocol was approved by the University of Melbourne Human Research Ethics Committee (Ethics ID 1238477). Participants gave verbal informed consent to study participation prior to administration of the telephone questionnaire.

\section{Results}

\section{Study population}

A total of 25,406 calls were made to 8567 numbers, of which 7129 were currently connected telephones in residential households (2755 of 3398 in Boroondara, 4374 of 5169 in Hume). Contact was made with an individual in 4580 of these households (1827 in Boroondara and 2753 in Hume), with a further 683 proving ineligible due to geographical location (out of area), communication difficulties or absence of an adult present at the time of call. Communication difficulties reported included language difficulty $(n=28)$ or other physical limitation such as hearing impairment or age $(n=131)$. Of the remaining 3897 eligible contacts, 1307 (650 in Boroondara, 657 in Hume) completed the survey, with 12 interviews conducted in Turkish. Responses were spread across days of the week such that the numbers are fairly even across weekdays (range: 191-211) and separately across weekends (range: 156-157). Response rates according to the 'all contacts' denominator were $36 \%(650 / 1827)$ in Boroondara and $24 \%$ (657/2753) in Hume. When calculated as all completed interviews over a denominator comprising completions, refusals and break-offs, the response rate overall was $37.6 \%$, remaining higher in Boroondara (46 \%) than Hume (32\%).

While the final number in the sample was less than our initial target of 2000, a pragmatic decision was made to cease recruitment at 1307 , given greater than anticipated time requirements per participant and finite budgetary constraints. Implications of study complexity related to the telephone interview format for missing and incomplete data are described in more detail below.

\section{Weighting}

Compared with 2011 Australian census data, characteristics of the sample differed significantly from those of the areas surveyed across a range of demographic and socio-economic factors in both Hume and Boroondara. Important differences included an over-representation of individuals who were aged over 50 years, female, Australian born, English speaking, educated to completion of secondary school, and married. In keeping with these characteristics, smaller households were overrepresented. The anticipated bias towards longer resident individuals was also observed. Given this disparity, weighted results are presented for all aggregated data, in order to present results more likely to be representative of the populations under study. For more details, see Sections 5 Biased sampling, raking and sampling weights and 6 Tables demonstrating bias in CATI data of Additional file 1. 


\section{Characteristics of encounters, by location}

Unique addresses visited by participants over the course of the survey day were categorised and distributed as shown in Fig. 2 (top left panel). All but six participants spent some time at their usual home, with retail/hospitality, and private transport being the next most common types of designated settings. Approximately one tenth of locations were assigned as 'other', of which about half were non-participant private homes, with the remainder comprising places such as medical centres and facilities, and places of worship. The weighted number of total listed encounters by location is reported in Fig. 2 (top right panel) (and Additional file 4: Figure S1 for physical contact). These data demonstrate the high median number of contacts made in school and daycare settings, but also the marked heterogeneity in encounter profiles within several location types including home, private transport, retail/hospitality and 'other'. Figure 2 (bottom left panel) highlights the greater length of interactions with workplace colleagues over the course of a working day, relative to those in most other domains. Figure 2 (bottom right panel) similarly reports duration of encounters, but restricted to those involving any physical contact. This last figure strongly reasserts the importance of household settings in providing opportunities for close-contact transmission of infection.

\section{Number and duration of social encounters, by age and gender}

Figure 3 (left panel) reports the unweighted total number of listed encounters for each participant in the survey, with a mean of 8.4 (95\% CI: 7.9-8.8) and median of 7 . The weighted total number of listed encounters for each participant across all participants (mean 8.5, 95 \% CI: 7.9-9.1) and across days of the week (mean range 8.0-9.3, median range 6-8) was similar. In addition, the distinction between weekend and weekday is not a significant predictor for the number of listed encounters $(p=0.9)$. Figure 3 (right panel) shows a boxplot of the weighted number of all encounters by age group. The
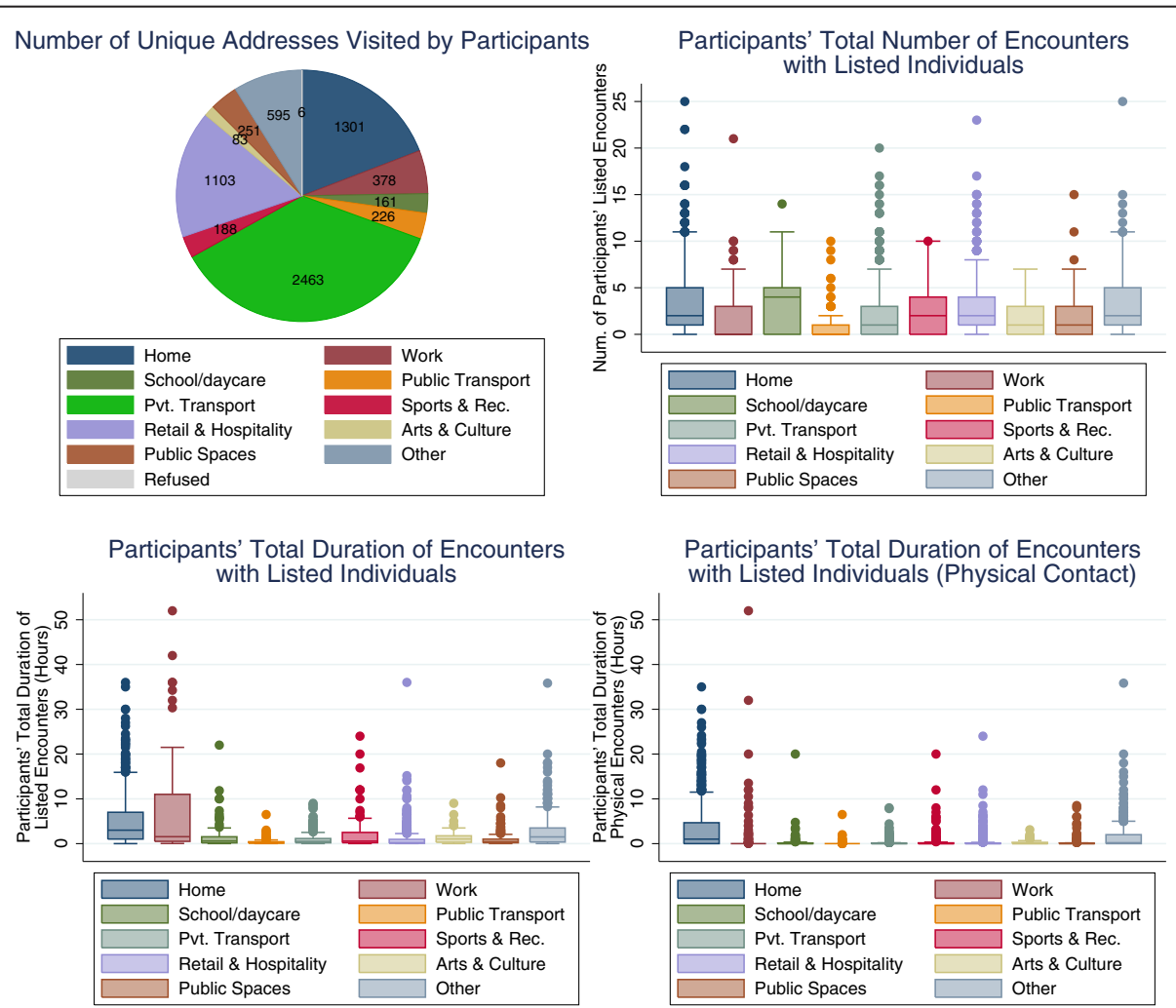

Fig. 2 Characteristics of locations and encounters by location type. Summaries of locations and encounters from one study day for each participant, reported separately for each location type. The number of unique addresses visited by participants (top left panel). Boxplot for participants' total (weighted) number of encounters with listed individuals, by location (top right panel). Boxplot for participants' total (weighted) duration of encounters with listed individuals, by location (bottom left panel). Boxplot for participants' total duration of encounters involving physical contact with listed individuals, by location (bottom right panel). For all boxplots, boxes denote the interquartile range, interior lines shown the median and the whiskers show adjacent values. Across the boxplots, marked heterogeneity is apparent in several location types. For total duration of contact, Work (and Home to a lesser degree) is the dominant location type. For total duration of physical contact, home is the dominant location type. Boxplots use raking weights to reduce the effects of sample bias. For each location type, participants with no time spent at that location type do not contribute to those results 

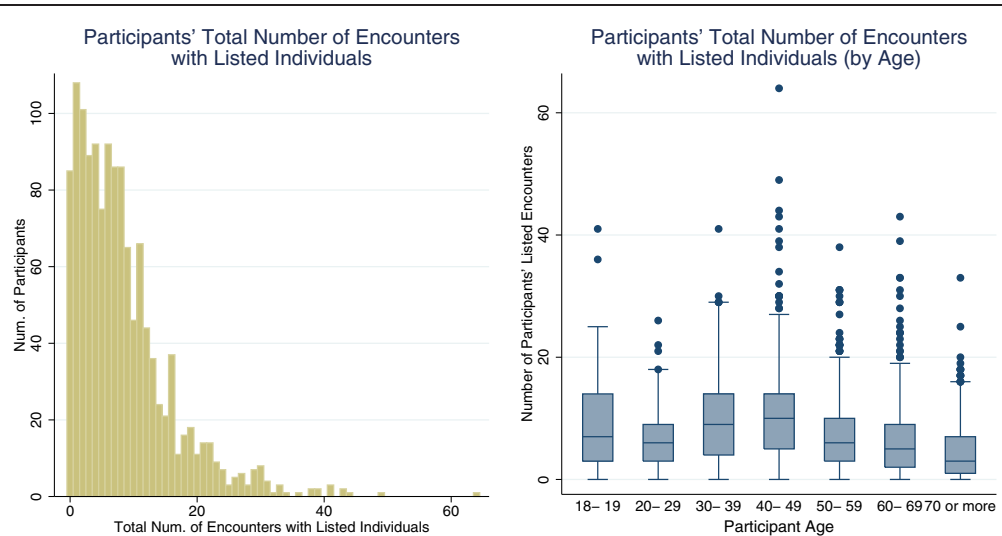

Fig. 3 Participants' total number of encounters with listed individuals. Histogram for participants' total (unweighted) number of encounters with listed individuals (left panel). Boxplot for participants' total (weighted) number of encounters with listed individuals by participant age (right panel). Heterogeneity is apparent across participants and across age groups. In general, participants' number of encounters declines with age. The boxplot uses raking weights to reduce the effects of sample bias

highest reported number of median contacts was among individuals aged between 30 and 49 years. This trend was more evident for episodes involving physical contact (Additional file 4: Figures S2 and S3).

Respondents frequently reported more than one encounter with the same individual over a $24 \mathrm{~h}$ period (Additional file 4: Figure S4). The weighted mean number of contacts (with $95 \%$ confidence intervals) between participants and uniquely nominated individuals is shown in Fig. 4. Results are reported across six participant age categories and ten contact age categories, for male (left panel) and female (right panel) respondents. The number of participants contributing to each cell varies, and both weighted and unweighted counts for each gender can be found in the supporting information (Additional file 4: Tables S1-S4 for all encounters and Tables S5S8 for physical encounters). While a generally assortative pattern of mixing is observed, we also note that females record many more encounters with children less than 15 years of age than males. Males aged 18-29, on the other hand, have the highest recorded withinage group interactions. Similar trends are observed for episodes involving any physical contact (Additional file 4: Figures S7 and S8). 
The duration of encounters, by age, is a further measure of mixing intensity, reported as mean hours (with $95 \%$ confidence intervals) in Fig. 5. Again, values for men and women are reported separately. The striking difference between this figure and Fig. 4 is the dominance of interactions between women in the 18-29 years group and children of pre-school age, most likely in household settings (Fig. 5- right panel). These prolonged mixing episodes are also associated with physical contact (Additional file 4: Figure S10 (right panel)). Encounters between males aged 18-29 years, previously noted to be both frequent and close, are also prolonged (Fig. 5 (left panel), and Additional file 4: Figure S10 (left panel) for physical contact).

Heterogeneity of encounters was further assessed by 692 reports of mixing with social groups of six people or more. The median age of participants reporting group contacts was 52 years (IQR 40, 62). Median reported group size was 12 (interquartile range 9,20 ), with $40 \%$ of such contacts occurring in the workplace. Retail and hospitality, sport and recreational settings, and educational environments each accounted for approximately $10 \%$ of listed group encounters. A large 'other' category included places of worship, clubs and private social gatherings. Mixing matrices for unweighted numbers of encounters and duration of encounters (in person-contact-hours) for participant contact made in a group setting are available in Additional file 4: Sections 1.10 and 1.11, where each group was categorised with one typical age (0-19 years, $20-69,70$ or more) by the participant. Given uncertain overlap between mixing groups and individually reported contacts, subsequent analyses report only on uniquely identified individuals.

\section{Encounters with known and unknown individuals}

Participants were asked to differentiate between encounters with individuals known to them and strangers. Young men, and women aged 30-49 years, reported many more known contacts than other respondents (Fig. 6 -left panel). In the former case, the majority of these contacts were non-household members, while among the women about half of the contacts involved family (Fig. 6 - middle). For both sexes, the total number of known contacts both inside and outside the home tended to increase with household size (Fig. 6 -bottom panel). (See Additional file 4: Figure S11 for participants' total duration of contacts by contact type and household size.) The vast majority (75 \%) of group contacts (see above) involved individuals known to participants.

\section{Influence of local government area of residence on encounter profiles}

Summary measures of encounters, as presented in the Figures above, were compared between the LGAs surveyed in an initial exploratory analysis. While social characteristics of participants in both regions were broadly similar, some suggestions of difference emerged, warranting further evaluation. Key findings are reported in Table 1, with caution recommended in the interpretation of estimates where sample sizes were $<60$. Boroondara (B) residents reported mixing with more people on average in public spaces than Hume $(\mathrm{H})$ residents, and

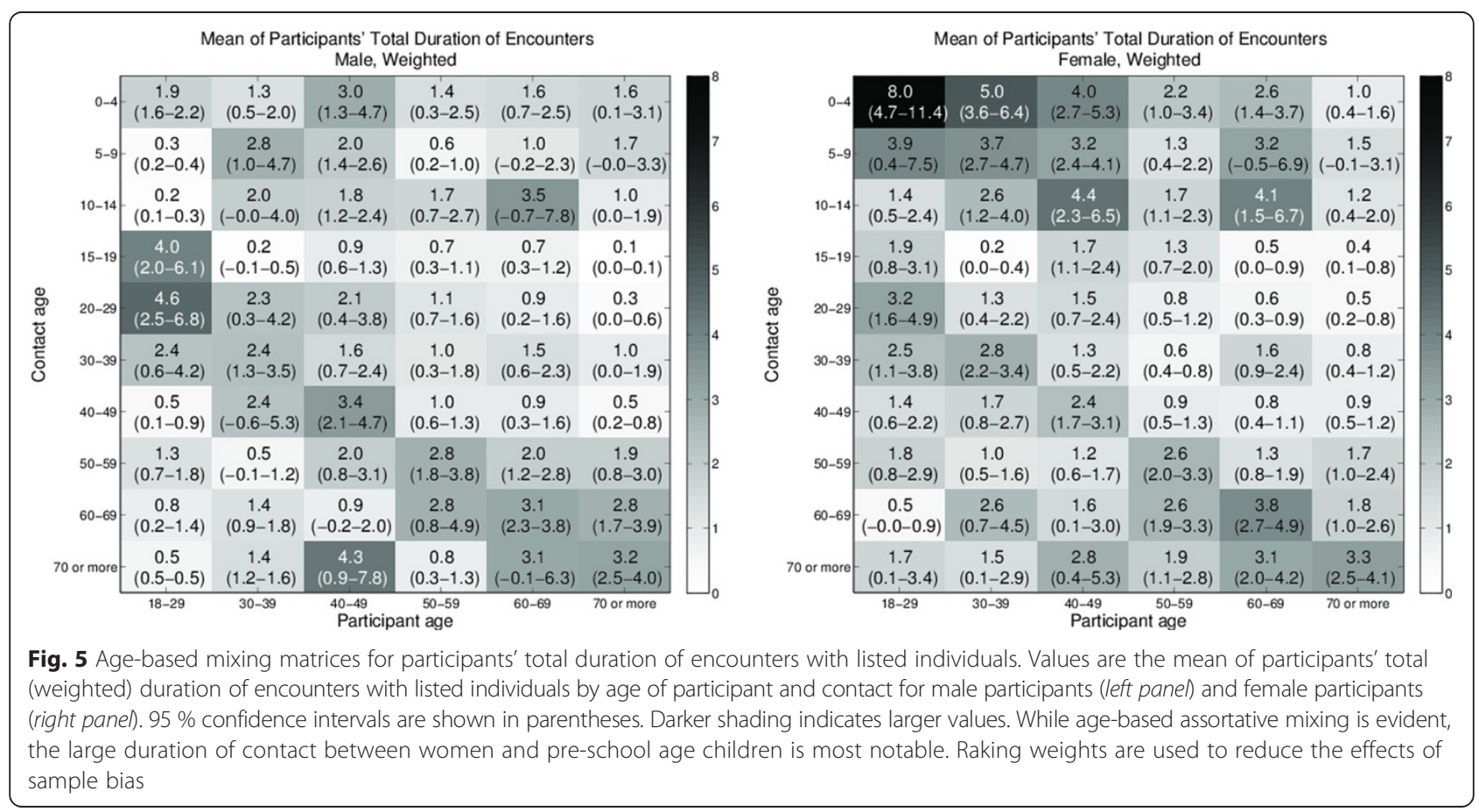



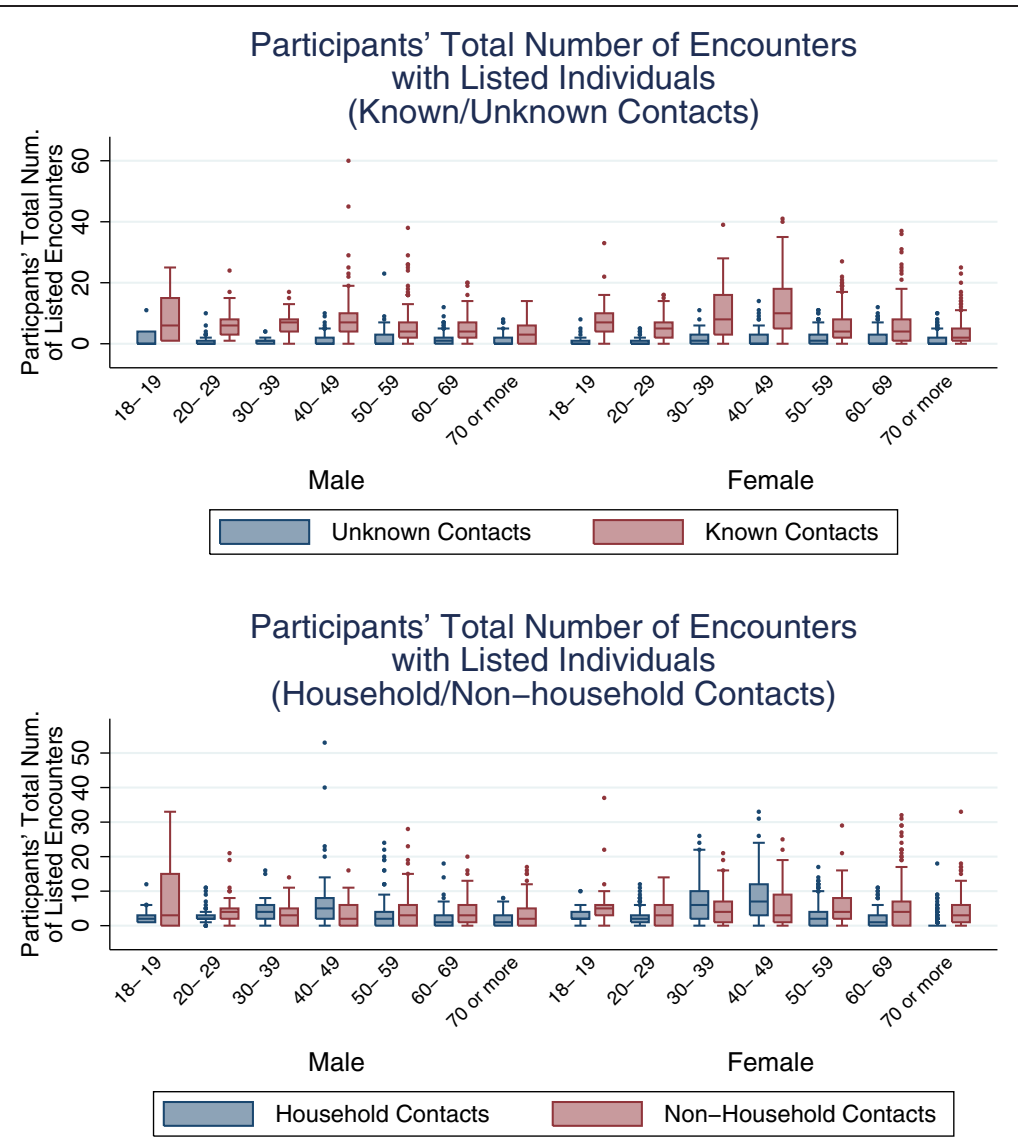
Participants' Total Num. of Encounters with Listed Individuals

(by household size, contact type, location)

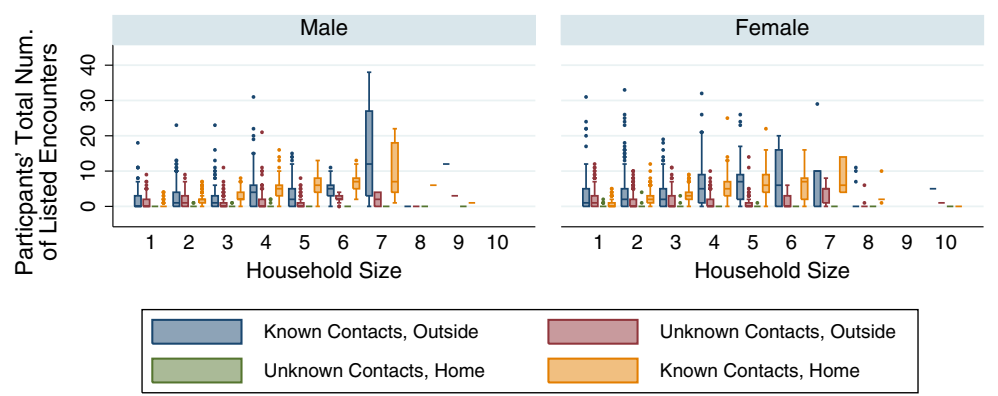

Fig. 6 Participants' total (weighted) number of listed encounters by type of contact individual. Boxplots for participants' total number of listed encounters by known/unknown contacts for male (left) and female (right) participants (top panel), household/non-household contacts for male (left) and female (right) participants (middle panel), and by household size, known/unknown contacts, and location of contact (Home/Outside) for male (left) and female (right) participants (bottom panel). Young men and women aged 30-49 years reported many more encounters with listed people than other participants. For young men, the majority are non-household members. For women, about half involved household members. In general, the number of encounters with known individuals increases with household size. Raking weights are used to reduce the effects of sample bias

similarly in retail and hospitality environments, of which more were visited [B: $1.8(1.6,2.0) \mathrm{cf} H: 1.4$ (1.3, 1.6), $p=0.007$ ]. Hume residents tended to spend longer periods of time (hours) with others, particularly in arts and culture settings, although this latter estimate was based on a small sample size [B: $15 \mathrm{cf} \mathrm{H}$ : 9].
Women less than 20 years of age in Hume had contact with twice as many known individuals than in Boroondara, based on a limited number of participant reports [B: $10 \mathrm{cf}$ H: 9]. Young men (20-29 years) in Hume socialised more with non-household members than those in Boroondara, although again, few participated in the study [B: $18 \mathrm{cf} \mathrm{H}$ : 
Table 1 Influence of local government area of residence on encounter profiles

\begin{tabular}{|c|c|c|c|}
\hline & $\begin{array}{l}\text { Boroondara mean } \\
(95 \% \mathrm{Cl})\end{array}$ & $\begin{array}{l}\text { Hume mean } \\
(95 \% \mathrm{Cl})\end{array}$ & $P$-value \\
\hline \multicolumn{4}{|l|}{ Number of listed encounters } \\
\hline Public spaces & $2.2(1.6-2.8)$ & $1.4(0.9-1.8)$ & 0.029 \\
\hline Retail \& hospitality & $3.3(2.9-3.7)$ & $2.7(2.3-3.0)$ & 0.013 \\
\hline \multicolumn{4}{|l|}{ Number of unique addresses } \\
\hline Retail \& hospitality & $1.8(1.6-2.0)$ & $1.4(1.3-1.6)$ & 0.007 \\
\hline \multicolumn{4}{|c|}{ Participants' total encounter duration (Hours) } \\
\hline Participant total & $6.2(5.5-6.9)$ & $7.5(6.6-8.4)$ & 0.028 \\
\hline \multicolumn{4}{|c|}{ Participants' total physical encounter duration (Hours) } \\
\hline Arts \& culture & $0.5(0.4-0.7)$ & $1.1(0.8-1.5)$ & $0.004^{*}$ \\
\hline \multicolumn{4}{|c|}{ Participants' total number of known contacts } \\
\hline Female 18-19 & $6.5(4.6-8.4)$ & $12.7(6.9-18.5)$ & $0.048^{*}$ \\
\hline \multicolumn{4}{|c|}{ Participants' total number of household contacts } \\
\hline Female 18-19 & $2.4(1.5-3.2)$ & $5.0(2.5-7.6)$ & $0.05^{*}$ \\
\hline \multicolumn{4}{|c|}{ Participants' total number of non-household contacts } \\
\hline Male, age $20-29$ & $2.9(1.7-4.2)$ & $5.2(3.7-6.8)$ & $0.025^{*}$ \\
\hline \multicolumn{4}{|c|}{ Participants' total number of unknown contacts } \\
\hline Female, age $50-59$ & $2.8(2.1-3.4)$ & $1.5(1.0-1.9)$ & 0.002 \\
\hline Female, age 60-69 & $2.1(1.5-2.7)$ & $1.2(0.6-1.8)$ & 0.027 \\
\hline Female, age 70 or more & $1.7(1.2-2.1)$ & $0.7(0.3-1.0)$ & $<0.001$ \\
\hline Male, age 60-69 & $1.9(1.2-2.6)$ & $0.9(0.5-1.4)$ & 0.022 \\
\hline
\end{tabular}

*Indicates both sample sizes are smaller than 60 , which may affect validity of the $p$ value

19]. At the other end of the age spectrum, contact with unknown individuals was more frequent in Boroondara than Hume among females aged 50-59 and older, and males from 60 years of age.

\section{Discussion}

Better data about patterns of social contact is needed to enable more accurate parameterisation of disease transmission models [15]. Ascertainment of conversational and physical encounters using a CATI format, while challenging to implement, yielded rich information across a large population sample. External factors known to influence mixing rates, including marked climatic variation [16], peak periods of respiratory illness [17] and school breaks $[18,19]$ were avoided by conducting the study over just a few months in summer and early autumn, and all in school term time. Within this context, marked heterogeneity of social behaviour was observed by location, age, gender and area of residence by attending to more detailed attributes of encounters including location, household membership and 'strangeness' than merely the number of contacts recorded.

Cost-efficiency was our primary motivation for trialling a CATI survey, allowing recruitment of a large and geographically well-defined population sample across two sites for this 'proof of concept' study. Limitations of the CATI approach included predictable biases in ascertainment [20], leading to an unrepresentative sample from each of the study areas (Additional file 1). Complexity associated with verbal recall made the interviews longer than anticipated. Participants also expressed more privacy concerns than in face-to-face interviews, resulting in incomplete or missing information in many records. In consequence, substantial time was committed to weighting, cleaning and augmenting data.

Despite these challenges, we obtained extremely detailed information on the interactions of individuals and the types of settings in which these occur. The distribution of all encounters across types of locations is similar to that reported in a variety of European countries in the POLYMOD study [2]. We did, however, note a much greater weighting of physical encounters towards the home environment than seen in that survey [2], predominantly influenced by reports of female participants. This finding should be considered in the context of related work comparing sensor and diary recordings, showing that contacts of longer duration are more likely to be recalled, and that females as a group are more accurate reporters of social encounter information [21]. It is unlikely that this difference reflects disparity in parenting behaviour between Europe and Australia, but may further relate to the nature of our diary instrument. While POLYMOD asked participants to estimate a single block of total time spent with a given individual, our respondents sequentially listed all locations and environments over the course of the day. It seems likely that this latter means of recall would allow for more accurate summation of repeat encounters, highly likely to occur in the household setting, and perhaps explaining the higher estimated proportion of household contacts.

While the overall mean number of contacts per individual was relatively low compared with a US telephone survey [20] and our own earlier work [5], this finding likely reflected the age distribution of the respondent population (Fig. 3 - right panel). When considered by age group, our observations accorded more closely with recent data from a postal and internet survey in the UK, and showed a steady decline with age [22]. While a similar decrease in sociability with age was observed in both urban and rural settings in China [23], this phenomenon is not universal. A study in rural Vietnam noted an increase in mixing rates among individuals over 40 years, persisting into older age [24]. It is not known whether such cultural differences persist after migration.

We observed far closer mixing of women with household members than men, perhaps contributing to reported differences in patterns of infection transmission. In a classic study of Haemophilus influenzae from the 1940s, eight 
times higher concordance of bacterial carriage was noted between mothers and their children than between fathers and children [25]. While times and social norms have changed substantially over the period, we recently found women to be more effective transmitters of infection than men [26], even in households where children were not present. While few studies report encounters by gender, women in a 2009 US survey reported more conversational interactions than men, although the setting in which these occurred was not stated [20]. Such disparity was not, however, observed in Vietnam suggesting that these differences cannot be universally assumed [24].

Encounter studies involving large numbers of participants have generally been targeted at whole-country level, including POLYMOD $(n=7290)$ which recruited across 8 EU countries [2], a 2010 study of almost 2000 Taiwanese residents [27] and a recently published UKwide survey of more than 5000 respondents [22]. In contrast, a North Carolina telephone survey of social encounters recruited almost 4000 participants from four pre-specified counties, but no specific rationale was given for their selection [20]. A large Chinese study recruited 1821 individuals across a geographical zone spanning urban and rural environments, to consider the influences of distance and population density on social interactions [23]. Our explicit strategy was to densely sample two diverse areas of one major city in Australia, to understand qualitatively reported small area socio-demographic and environmental influences on social network heterogeneity [7]. The preliminary analyses presented here suggest varying location preferences, and gendered differences in mixing beyond the household unit, by area of residence, that will be explored in more detail in subsequent work. In particular, the geographical extent of social networks, and the overarching influences of both household and area level advantage, are of interest.

\section{Conclusions}

Mixing matrices based on physical encounter data such as these have been incorporated in age-structured transmission models, and validated through their ability to reproduce age-dependent infection profiles in cross-sectional population serosurveys [28]. More recently, encounter measures have also been correlated with laboratory confirmed evidence of respiratory infection at the individual level [29, 30]. Assumptions regarding the number, duration and clustering of contacts in model frameworks all have significant implications for the simulated spread of infection [31], and optimal strategies for its control [3]. Our hypothesis is that differences in social behaviour may contribute, at least in part, to the increasing infection risk observed with disadvantage [32], mediated at the level of the household, neighbourhood or workplace [33].
The incorporation of geographical space into representations of social networks is a recognised challenge in the field of infectious disease modelling [34]. As diversity increases in modern cities [35], location of residence encodes a far greater variety of attributes that may influence social behaviour than merely distance between individuals, or proximity to mixing locations. Spatial differences in culture, advantage and environment will likely exert pronounced effects on network characteristics, with local implications for infectious disease transmission and risk. Further evaluation of this dataset will involve detailed profiling of small areas, to seek additional evidence of both household and neighbourhood level influences on social behaviour and development of network models of infection spread, to investigate the likely implications of difference for disease.

\section{Additional files}

Additional file 1: Cleaning, Geocoding and Weighting. Additional details on data cleaning, geocoding of addresses, sample bias and sample weighting using raking. (PDF $900 \mathrm{~kb}$ )

Additional file 2: Questionnaire. Additional file descriptions text (including details of how to view the file, if it is in a non-standard format). (PDF $130 \mathrm{~kb}$ )

Additional file 3: Contact Diary. Additional file descriptions text (including details of how to view the file, if it is in a non-standard format). (PDF $61 \mathrm{~kb}$ )

Additional file 4: Additional Results. Additional results including details on number and duration of physical contact encounters, and duration of encounters for each household size by contact type (known/unknown) and location type (home/outside). (PDF 1275 kb)

\section{Competing interests}

The authors have no competing interests to declare.

\section{Authors' contributions}

$N G, D W, G R, P P, J M C M$ and JMCV contributed to the design of the study. DW, $P N, G R, J M C M$ and JMCV were involved in developing survey materials. DR cleaned the data and undertook all analyses, in consultation with NG, JMcM and JMCV. All authors were engaged in drafting the manuscript and have seen and approved the final version.

\section{Acknowledgements}

This project was supported by an ARC Linkage Project grant, with contributing partners VicHealth, the Victorian Government Department of Health, the City of Boroondara, and Lentara Uniting Care. We further acknowledge fellowship funding for NG (ARC Discovery Early Career Researcher Fellowship), DW and JMM (ARC Future Fellowships) and JMV (NHMRC Career Development Fellowship).

We are grateful to our participants, for sharing their information through the telephone survey, and to the Social Research Centre for their skill and diligence in assisting us with a challenging protocol. We thank Rebecca Bentley for helpful comments on the manuscript.

\section{Author details}

${ }^{1}$ Melbourne School of Psychological Sciences, The University of Melbourne, Melbourne, Australia. ${ }^{2}$ Modelling and Simulation Unit, Centre for Epidemiology and Biostatistics, Melbourne School of Population and Global Health, The University of Melbourne, Melbourne, Australia. ${ }^{3}$ McCaughey VicHealth Community Wellbeing Unit, Melbourne School of Population and Global Health, The University of Melbourne, Melbourne, Australia. ${ }^{4}$ School of Mathematics and Statistics, The University of Melbourne, Melbourne, Australia. ${ }^{5}$ Modelling and Simulation Unit, Infection and Immunity Theme, Murdoch Childrens Research Institute, Parkville, Australia. 
Received: 28 April 2015 Accepted: 20 October 2015

Published online: 02 November 2015

\section{References}

1. Ball F, Britton F, House T, Isham V, Mollison D, Pellis L, et al. Seven challenges for metapopulation models of epidemics, including households models. Epidemics. 2014. In press.

2. Mossong J, Hens N, Jit M, Beutels P, Auranen K, Mikolajczyk R, et al. Social contacts and mixing patterns relevant to the spread of infectious diseases. PLoS Med. 2008;5(3), e74

3. Salathe M, Jones JH. Dynamics and control of diseases in networks with community structure. PLoS Comput Biol. 2010;6(4), e1000736.

4. Perkins TA, Garcia AJ, Paz-Soldan VA, Stoddard ST, Reiner RC Jr, VazquezProkopec G, et al. Theory and data for simulating fine-scale human movement in an urban environment. J R Soc Interface. 2014, 11(99). doi:10.1098/rsif.2014.0642.

5. McCaw JM, Forbes K, Nathan PM, Pattison PE, Robins GL, Nolan TM, et al Comparison of three methods for ascertainment of contact information relevant to respiratory pathogen transmission in encounter networks. BMC Infect Dis. 2010;10:166.

6. Bolton KJ, McCaw JM, Forbes K, Nathan P, Robins G, Pattison P, et al. Influence of contact definitions in assessment of the relative importance of social settings in disease transmission risk. PLoS One. 2012;7, e30893.

7. Warr D. Social networks in a 'discredited' neighbourhood. J Sociol. 2005;41(3):285-308.

8. Australian Bureau of Statistics. 2011 Census of Population and Housing: Basic Community Profile (Hume); 2012.

9. Australian Bureau of Statistics. 2011 Census of Population and Housing: Basic Community Profile (Boroondara); 2012.

10. Locations API. [http://msdn.microsoft.com/en-us/library/ff701715.aspx].

11. MapQuest Platform Web Services. [http://www.mapquestapi.com/].

12. Nominatim - OpenStreetMap Wiki. [http://wiki.openstreetmap.org/wiki/ Nominatim].

13. Google Maps. [http://maps.google.com.au/maps].

14. TableBuilder. [http://www.abs.gov.au/websitedbs/censushome.nsf/home/ tablebuilder].

15. Riley S, Eames KTD, Isham V, Mollison D, Trapman P. Five challenges for spatial epidemic models. Epidemics. 2014. In press.

16. Chan T-C, Fu Y-C, Hwang J-S. Changing social contact patterns under tropical weather conditions relevant for the spread of infectious diseases. Epidemiol Infect. 2014;143:1-12.

17. Van Kerckhove K, Hens N, Edmunds WJ, Eames KTD. The impact of illness on social networks: implications for transmission and control of influenza. Am J Epidemiol. 2013;178:1655-62.

18. Eames KTD, Tilston NL, Edmunds WJ. The impact of school holidays on the social mixing patterns of school children. Epidemics. 2011;3:103-8.

19. Chen S-C, You Z-S. Social contact patterns of school-age children in Taiwan: comparison of the term time and holiday periods. Epidemiol Infect. 2014;143:1139-47.

20. DeStefano F, Haber M, Currivan D, Farris T, Burrus B, Stone-Wiggins B, et al. Factors associated with social contacts in four communities during the 2007-2008 influenza season. Epidemiol Infect. 2011;139:1181-90.

21. Smieszek T, Barclay VC, Seeni I, Rainey JJ, Gao H, Uzicanin A, et al. How should social mixing be measured: comparing web-based survey and sensor-based methods. BMC Infect Dis. 2014;14:136

22. Danon L, Read JM, House TA, Vernon MC, Keeling MJ. Social encounter networks: characterizing Great Britain. Proc Biol Sci. 2013;280:20131037.

23. Read JM, Lessler J, Riley S, Wang S, Tan LJ, Kwok KO, et al. Social mixing patterns in rural and urban areas of southern China. Proc Biol Sci. 2014;281:20140268

24. Horby P, Pham QT, Hens N, Nguyen TTY, Le QM, Dang DT, et al. Social contact patterns in Vietnam and implications for the control of infectious diseases. PLoS One. 2011;6, e16965.

25. Boisvert PL. Familial epidemiology of Hemophilus influenzae, type B, infections. Am J Dis Child. 1948;75(3):427.

26. McCaw JM, Howard PF, Richmond PC, Nissen M, Sloots T, Lambert SB, et al. Household transmission of respiratory viruses - assessment of viral, individual and household characteristics in a population study of healthy Australian adults. BMC Infect Dis. 2012;12:345.

27. Fu Y-C, Wang D-W, Chuang J-H. Representative contact diaries for modeling the spread of infectious diseases in Taiwan. PLoS One. 2012;7:e45113.
28. Melegaro A, Jit M, Gay N, Zagheni E, Edmunds WJ. What types of contacts are important for the spread of infections?: using contact survey data to explore European mixing patterns. Epidemics. 2011;3:143-51.

29. Kucharski AJ, Kwok KO, Wei WWI, Cowling BJ, Read JM, Lessler J, et al. The contribution of social behaviour to the transmission of influenza $A$ in a human population. PLoS Pathog. 2014;10:e1004206.

30. Kwok KO, Cowling BJ, Wei WWI, Wu KM, Read JM, Lessler J, et al. Social contacts and the locations in which they occur as risk factors for influenza infection. Proc Biol Sci. 2014;281:20140709.

31. Danon L, House TA, Read JM, Keeling MJ. Social encounter networks: collective properties and disease transmission. J R Soc Interface. 2012;9(76):2826-33.

32. Balasegaram S, Ogilivie F, Glasswell A, Anderson C, Cleary V, Turbitt D, et al Patterns of early transmission of pandemic influenza in London - link with deprivation. Influenza Other Respi Viruses. 2012;6(3):e35-41.

33. Kumar S, Quinn SC, Kim KH, Daniel LH, Freimuth VS. The impact of workplace policies and other social factors on self-reported influenza-like illness incidence during the 2009 H1N1 pandemic. Am J Public Health. 2012;102(1):134-40.

34. Read JM, Edmunds WJ, Riley S, Lessler J, Cummings DA. Close encounters of the infectious kind: methods to measure social mixing behaviour. Epidemiol Infect. 2012;140(12):2117-30.

35. Vertovec S. Super-diversity and its implications. Ethnic Racial Stud. 2007;30(6):1024-54.

36. QGIS software v2.6: QGIS Development Team, 2014. Open Source Geospatial Foundation Project. In.

37. ABS LGA Map Data. Australian Bureau of Statistics 2011, Statistical Geography Standard (ASGS): Volume 3 - Non ABS Structures, July 2011, data cube cat. no. 1270.0.55.003, viewed 10 Dec 2014.

38. VicMap Built up areas: State Government of Victoria, Statewide Place Area boundaries, polygon - 1:250,000 to 1:5 million, http://www.data.vic.gov.au, licensed under CC BY $3.0 \mathrm{AU}$ (http://creativecommons.org/licenses/by/3.0/ au/deed.en), viewed 10 Dec 2014

\section{Submit your next manuscript to BioMed Central and take full advantage of:}

- Convenient online submission

- Thorough peer review

- No space constraints or color figure charges

- Immediate publication on acceptance

- Inclusion in PubMed, CAS, Scopus and Google Scholar

- Research which is freely available for redistribution 